\title{
Improved linearised models of wind turbine aerodynamics and control system dynamics using harmonic linearisation
}

\author{
Richard C Lupton ${ }^{\mathrm{a}, *}$, Robin S Langley ${ }^{\mathrm{a}}$ \\ ${ }^{a}$ Department of Engineering, University of Cambridge, Trumpington St, Cambridge, CB2 1PZ, UK
}

\begin{abstract}
Where non-linearities are not too strong, linearised frequency-domain approaches offer fast calculations, which can be valuable for preliminary design of wind turbine blades, foundations and floating platforms. But the aerodynamic and control system behaviour of a wind turbine is noticeably non-linear. Here we show for the first time that the technique of harmonic linearisation can reduce error in the approximation of aerodynamic and control system non-linearities, compared to the more common tangent linearisation. After deriving the linearised models, comparing linearised results to non-linear simulations for the NREL 5MW turbine shows that: (1) harmonic linearisation captures aero-elastic effects and non-linearity in aerodynamic forces, giving a 2-4x reduction in error compared to the tangent linearisation; (2) harmonic linearisation can capture non-linear wake dynamics; and (3) the torque and pitch controller behaviour can be approximated with good results away from the rated wind speed but with some challenges when the two controllers interact. Further improvements in the linearised model of the control system have been identified. By improving the accuracy of linearised models, harmonic linearisation is a promising means to extend the applicability of frequency-domain approaches for initial design and optimisation of wind turbines.
\end{abstract}

Keywords: wind energy, aerodynamic loads, frequency-domain modelling, harmonic linearisation, equivalent linearisation, non-linearity

\section{Introduction}

There are many sources of non-linearity in wind turbines. Some non-linearities can reasonably be neglected for some purposes, such as structural non-linearity [1] and secondorder hydrodynamic forces [2]. But some are more significant, in particular the aerodynamic loads and the control system dynamics. For example, Figure 1 shows the thrust curve of part of a wind turbine rotor, which is clearly non-linear when moderately large variations in wind speed are considered, and Figure 2 shows examples of non-linearity in the torque controller response.

Non-linearities are important because they determine the choice of modelling methods. When non-linearities are not too dominant, linearised frequency-domain approaches give fast calculation of loading and response spectra and statistics. Although generally useful, this is particularly valuable for analysing floating wind turbines, where the frequencydomain approach is well established for analysis of other floating structures, and a large number of load cases arise from the possible combinations of sea and wind states. Linearised methods have been used for modelling stall-regulated turbines [3, 4], offshore

\footnotetext{
*Corresponding author

Email addresses: rc133@cam.ac.uk (Richard C Lupton), rsl21@cam.ac.uk (Robin S Langley)
} 

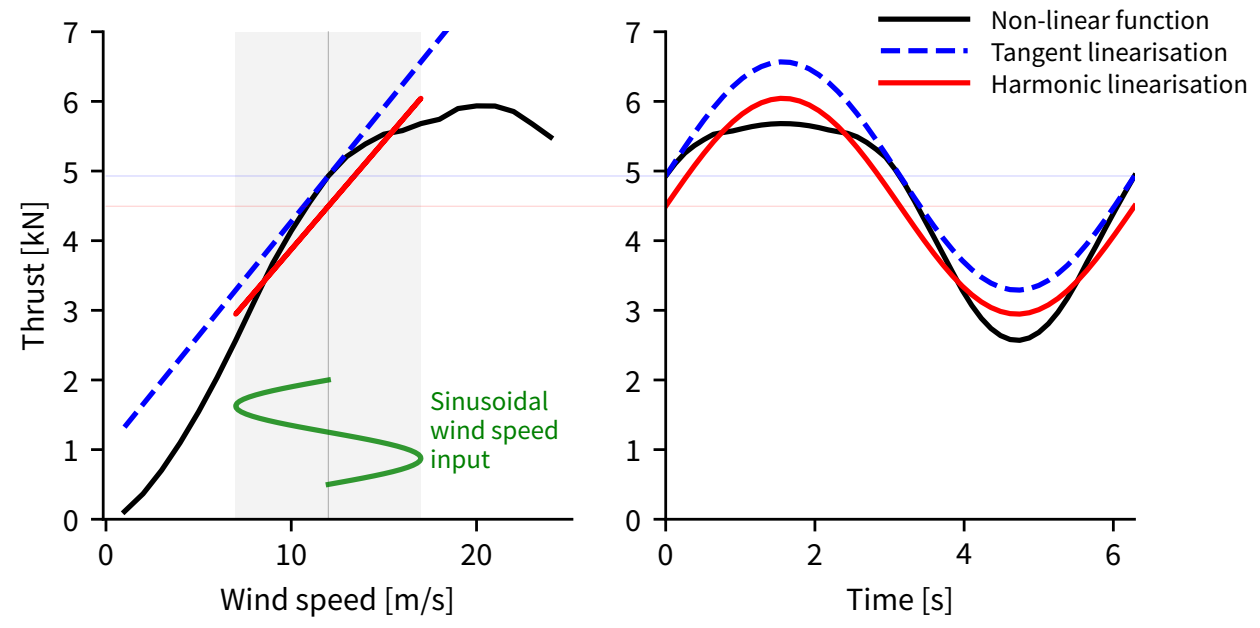

Figure 1: Non-linearity is significant in the aerodynamic loading on a wind turbine. This example shows the thrust on one rotor annulus, calculated from a Blade Element Momentum (BEM) model. The tangent and harmonic linearisations of the thrust are shown, with all inputs held constant apart from a sinusoidal variation in wind speed. Left: as a function of wind speed. Right: as a function of time.

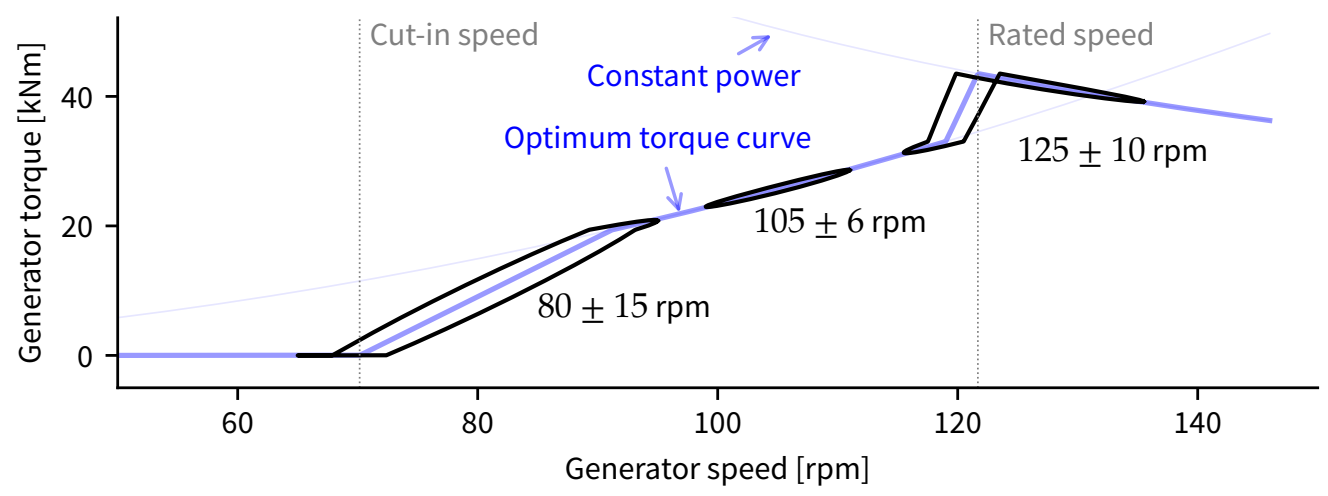

Figure 2: Examples of the response of the torque controller to harmonic variations in generator speed. The example at the left is mildly non-linear at the corners of the torque curve. For the example in the middle the underlying behaviour is quadratic, but over the range shown it is well approximated by the linear solution. The example at the right is highly non-linear. 
turbines [5], and initial design of foundations [6] and blades [7]. For floating turbines, they have been used to study a wide space of possible concepts [8] and to test the effect of wave energy converters on spar platforms [9]. More generally, linearised models are frequently used as a starting point for controller design [10].

However, the behaviour shown in Figure 1 is significantly non-linear, which calls into question whether linearised methods provide sufficient accuracy for modelling wind turbines. Halfpenny [3] found that non-linear aerodynamic forces were the main source of errors in his frequency-domain analysis of a stall-regulated turbine. Sabale and Gopal [11] highlight further non-linear effects using a geometrically-exact beam model accounting for aero-elasticity. Kvittem and Moan [12] compared frequency- and time-domain models of tower-bending moments in a floating wind turbine, finding that wind-induced low-frequency bending moments were not captured well, attributing this to lost non-linear thrust and the use of an aerodynamic damping model for a fixed turbine. Philippe et al. [13] compared frequency-domain and time-domain simulations of a floating wind turbine, but focused on the hydrodynamic loads. Generally, non-linear time-domain simulations are used for modelling wind turbines, which gives greater accuracy than linearised methods at the expense of greater simulation time. In this paper we ask if there is another way: can we better capture the aerodynamic and control system non-linearity of a wind turbine, to improve accuracy of loads and deflections while retaining the benefits of the frequency-domain approach?

Previous linearised models are mostly derived by perturbing a numerical non-linear model, for example using the codes FAST [14] or Bladed [15], or by analytical linearisation of the aerodynamic forces [16], giving a "tangent linearisation". Olondriz et al. [17] presents an alternative linearisation method using a "chirp" signal in FAST. Merz et al. [4] showed that, with ingenuity, linearised models can do better than this, concluding that while the results of their linearised aerodynamic model of a stall-regulated turbine were not good enough for certification, they were ideal for preliminary design and optimisation. In this paper we propose to use equivalent linearisation, also called harmonic linearisation and stochastic linearisation when used with harmonic and random inputs respectively. This aims to find an equivalent linear system which is in some sense the optimum approximation to the real function, given the inputs which actually occur. Specifically, the mean-squared error between the non-linear and linear functions is minimised [18]. For example, Figure 1 shows the harmonic linearisation of the thrust force for a sinusoidal variation in wind speed. There is a limit to how well a single sinusoid can be made to fit the output of the original non-linear function, but the harmonic linearisation gives the best possible sinusoid. This method is used to linearise the non-linear drag forces on submerged structures [19] but to our knowledge, this is the first time that harmonic linearisation has been demonstrated to improve the linearisation of aerodynamic loads and control system behaviour in modelling the loads and deflections of wind turbines.

Specifically, the contributions of this paper are as follows. The harmonic linearisation of the aerodynamic forces on a wind turbine rotor is derived, accounting for structural dynamics and aero-elasticity, wake dynamics and active control of the rotor speed and blade pitch angle (Sections 2-3). The results of the harmonic and tangent linearisations are then compared to non-linear reference results for the NREL 5MW turbine [20], for a range of wind conditions, focusing on three sources of non-linearity in turn:

1. aero-elastic effects and non-linearity in aerodynamic forces (Section 4);

2. non-linear wake dynamics (Section 5); and

3. the torque and pitch controller behaviour (Section 6).

The focus is on harmonic linearisation, but Section 7 discusses how a similar approach applies to stochastic inputs, and concludes by discussing the relevance of these results for the linearised modelling of the dynamic response of wind turbines. 


\section{Model setup and linearisation}

First the non-linear equations are set up, which govern the response of a wind turbine to aerodynamic loads, with a dynamic wake, and variable rotor speed and blade pitch angle. The tangent and harmonic linearisations which are used in the rest of the paper are then introduced.

In this model, the dynamic response of the wind turbine to aerodynamic loads can be described by the system equations:

$$
\begin{aligned}
& \text { Structural response: } \quad \boldsymbol{M} \ddot{\boldsymbol{q}}+\boldsymbol{C} \dot{\boldsymbol{q}}+\boldsymbol{K} \boldsymbol{q}=\boldsymbol{F}_{\text {aero }} \\
& \text { Rotor dynamics: } \\
& \text { Dynamic wake: } \\
& J \dot{\Omega}=Q_{\text {aero }}-G Q_{g}\left(\Omega_{g}\right) \\
& \dot{\mathbf{u}}=g\left(U_{\infty}, \mathbf{u}\right) \\
& \text { Control system states: } \\
& \dot{\Omega}_{g}=\omega_{c}\left(G \Omega-\Omega_{g}\right) \\
& \dot{I_{\epsilon}}=\Omega_{g}-\Omega_{\text {rated }}
\end{aligned}
$$

The first equation describes the structural dynamic response to loading in terms of the modal amplitudes $\boldsymbol{q}$, including the floating platform motion if relevant. The structural mass matrix $\boldsymbol{M}$, damping matrix $\boldsymbol{C}$ and stiffness matrix $\boldsymbol{K}$ can be obtained from a finiteelement beam model. The second equation describes the dynamics of the rotor speed $\Omega$. The aerodynamic forces $\boldsymbol{F}_{\text {aero }}$ and overall rotor torque $Q_{\text {aero }}$ consist of the distributed lift and drag forces along the blades, $Q_{g}$ is the generator torque controlled to regulate the rotor speed and power output of the turbine, and $G$ is the gearbox ratio. The third equation describes the dynamic wake response, in which the flow around the rotor takes some time to react to changes in loading. The fourth and fifth equations describe control system states, the filtered generator speed $\Omega_{g}$ (with filter corner frequency $\omega_{c}$ ) and the pitch controller integral $I_{\epsilon}$. Each of these are described in the rest of this section.

\subsection{Aerodynamic loads}

In Equations (1a) and (1b), the aerodynamic forces $\boldsymbol{F}_{\text {aero }}$ and $Q_{\text {aero }}$ consist of the distributed lift and drag forces along the blades, which are assumed to be divided into $N$ independent annuli with the forces calculated from a Blade Element Momentum (BEM) model:

$$
\begin{aligned}
& \boldsymbol{F}_{\text {aero }}=\sum_{k=1}^{N} f^{k}\left(U_{\infty}, \Omega, \theta, u^{k}, u^{\prime k}, v_{x}^{k}, v_{y}^{k}\right) \\
& Q_{\text {aero }}=\sum_{k=1}^{N} Q^{k}\left(U_{\infty}, \Omega, \theta, u^{k}, u^{\prime k}, v_{x}^{k}, v_{y}^{k}\right)
\end{aligned}
$$

The annuli forces can be considered in isolation and then later superimposed because they are coupled only through the linear structural dynamics on the left of Equations (1a) and (1b). The forces depend non-linearly on three global variables - the wind speed $U_{\infty}$, the rotor speed $\Omega$ and the blade pitch angle $\theta$ - and four variables relating to an individual annulus $k$ - the induced velocities $u^{k}$ and $u^{\prime k}$, and the in-plane and out-of-plane blade velocities $v_{x}^{k}$ and $v_{y}^{k}$.

There are two main types of unsteady aerodynamics relevant to floating wind turbines: unsteady aerofoil aerodynamics, and the wake dynamics. The unsteady aerofoil behaviour is relatively high frequency, typically with periods of less than one second [21], and describes the delay between the flow conditions changing and a change in the lift and drag forces. The wake dynamics take place over longer periods related to the wind speed 


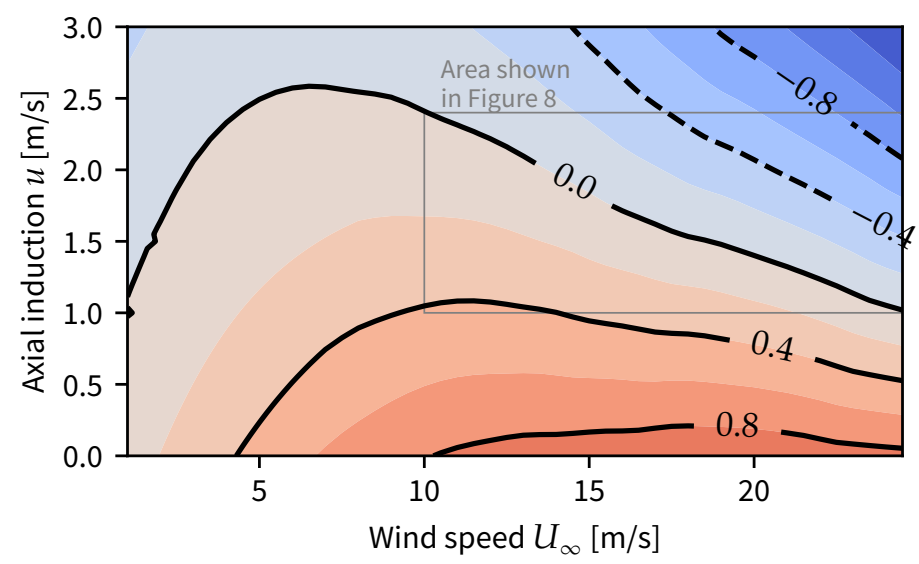

Figure 3: Wake derivative function $\dot{u}$ for the NREL blade. Rotor speed $9.45 \mathrm{rpm}$, pitch angle $0^{\circ}$, blade station at $43.7 \mathrm{~m}$.

and rotor dimensions, typically $5 \mathrm{~s}$ to $20 \mathrm{~s}$, and relate to the delay between a change in rotor loading and the change in flow speed seen at the rotor (the induced velocities $u$ and $\left.u^{\prime}\right)$. Here the focus is on the wake dynamics, using the dynamic wake model used by Bladed (Pitt and Peters 1981, reported by [15]), which defines the function $g\left(U_{\infty}, u\right)$ in Equation (1c), illustrated in Figure 3. The induced tangential velocity $u^{\prime}$ is neglected for simplicity, but could be included in an analogous manner.

Details of the aerodynamic model and verification are given in Lupton [22].

\subsection{Control system}

The aim of the torque controller is to maintain the optimum rotor speed which leads to the correct air flow for maximum aerodynamic efficiency, which is achieved by a quadratic relationship between rotor speed and generator torque [10, chapter 8]:

$$
Q_{g}=k_{\mathrm{opt}} \Omega_{g}^{2}
$$

The torque demand is based on the the filtered generator speed $\Omega_{g}$, governed by Equation (1d). In practice the optimum quadratic control can only be achieved over a limited range of generator speeds, and at the minimum and maximum generator speeds, the torque transitions linearly to zero and the rated generator torque respectively (Figure 2).

Once the rated power is being produced, the torque controller switches to constant power mode, as shown in Figure 2, and the pitch controller becomes active. To prevent the controllers conflicting, the torque controller is forced into constant power mode whenever the pitch angle is greater than some minimum value $\theta_{\mathrm{CP}}$ :

$$
Q_{g}= \begin{cases}f\left(\Omega_{g}\right) & \text { when } \theta \leq \theta_{\mathrm{CP}} \\ P_{\text {rated }} / \Omega_{g} & \text { otherwise }\end{cases}
$$

where $f\left(\Omega_{g}\right)$ is the function shown in Figure 2 .

The pitch controller is based on a PID controller acting on the error between the filtered generator speed $\Omega_{g}$ and the nominal rated generator speed $\Omega_{\text {rated }}$. The demanded pitch angle is

$$
\theta=G_{K}\left[K_{P}\left(\Omega_{g}-\Omega_{\text {rated }}\right)+K_{I} I_{\epsilon}\right]
$$

$K_{P}$ and $K_{I}$ are the proportional and integral gains respectively. The factor $G_{K}$ represents a 'gain schedule', which compensates for the variable sensitivity of the blade loads to 
changes in pitch angle at different wind speeds, determined by the pitch angle:

$$
G_{K}=\frac{1}{1+\theta / \theta_{2}}
$$

where $\theta_{2}$ is the pitch angle at which the gain should be halved. The pitch controller is automatically deactivated when $\Omega_{g}<\Omega_{\text {rated }}$ because then the error is negative, forcing the pitch angle to zero and preventing conflict with the torque controller.

\section{Tangent and harmonic linearisation}

This model of a wind turbine includes four non-linear functions that must be linearised to use a frequency-domain approach: the aerodynamic loads $\boldsymbol{F}_{\text {aero }}$ (Equation $1 \mathrm{a}$ ) and $Q_{\text {aero }}$ (Equation 1b), the wake dynamics $\dot{u}$ (Equation 1c), and the generator torque function $Q_{g}$ (Equation $1 \mathrm{~b}$ ). The tangent and harmonic linearisation approaches are now introduced for a general non-linear function $f(\mathbf{x}, \dot{\mathbf{x}})$, before applying them to the non-linear wind turbine model in the following sections.

In both cases harmonic variations in inputs at frequency $\omega$ are considered, which can be conveniently written in terms of complex exponentials as:

$$
\boldsymbol{x}(t)=\boldsymbol{x}_{0}+\frac{1}{2}\left(\overline{\boldsymbol{x}} e^{i \omega t}+\overline{\boldsymbol{x}}^{*} e^{-i \omega t}\right)
$$

where $\boldsymbol{x}_{0}$ is the mean value of $\boldsymbol{x}, \overline{\boldsymbol{x}}$ is a complex vector representing the magnitude and phase of $\boldsymbol{x}$, and $\overline{\boldsymbol{x}}^{*}$ is its complex conjugate. The non-linear response is not necessarily harmonic, but can be written similarly as:

$$
f(t)=f_{0}+\frac{1}{2}\left(\bar{f} e^{i \omega t}+\bar{f}^{*} e^{-i \omega t}\right)+\boldsymbol{\epsilon}(t)
$$

where $\boldsymbol{\epsilon}(t)$ represents higher harmonics in $f(t)$ at frequency $n \omega, n>1$, which are neglected in the linearised model. The difference between the tangent and harmonic linearisations lies in how the $f_{0}$ and $\bar{f}$ terms are calculated.

\subsection{Tangent linearisation}

The tangent linearisation is found by applying small perturbations $h$ about an operating point $\boldsymbol{x}_{0}, \dot{\boldsymbol{x}}_{0}$ as inputs to the non-linear function. The perturbed results are used to calculate the tangent stiffness and damping matrices:

$$
\begin{aligned}
& {\left[K_{f}\right]_{i j}=\frac{f_{i}\left(\boldsymbol{x}_{0}+\boldsymbol{h}_{j}, \dot{\boldsymbol{x}}_{0}\right)-f_{i}\left(\boldsymbol{x}_{0}-\boldsymbol{h}_{j}, \dot{\boldsymbol{x}}_{0}\right)}{2 h}} \\
& {\left[C_{f}\right]_{i j}=\frac{f_{i}\left(\boldsymbol{x}_{0}, \dot{\boldsymbol{x}}_{0}+\boldsymbol{h}_{j}\right)-f_{i}\left(\boldsymbol{x}_{0}, \dot{\boldsymbol{x}}_{0}-\boldsymbol{h}_{j}\right)}{2 h}}
\end{aligned}
$$

where $\left[\boldsymbol{h}_{j}\right]_{k}=h$ when $j=k, 0$ otherwise. The linearised approximation to $\boldsymbol{f}(\boldsymbol{x}, \dot{\boldsymbol{x}})$ is then

$$
\boldsymbol{f}(\boldsymbol{x}, \dot{\boldsymbol{x}}) \approx \boldsymbol{f}\left(\boldsymbol{x}_{0}, \dot{\boldsymbol{x}}_{0}\right)+\boldsymbol{K}_{f}\left(\boldsymbol{x}-\boldsymbol{x}_{0}\right)+C_{f}\left(\dot{\boldsymbol{x}}-\dot{\boldsymbol{x}}_{0}\right)
$$

Substituting in the harmonic input from Equation (7) gives:

$$
\begin{aligned}
& \boldsymbol{f}_{0}^{\tan }=\boldsymbol{f}\left(\boldsymbol{x}_{0}, \dot{\boldsymbol{x}}_{0}\right) \\
& \overline{\boldsymbol{f}}^{\mathrm{tan}}=\left(\boldsymbol{K}_{f}+i \omega \boldsymbol{C}_{f}\right) \overline{\boldsymbol{x}}
\end{aligned}
$$




\subsection{Harmonic linearisation}

In the harmonic linearisation, the mean and first harmonic of $f$ give the linearised coefficients [23]:

$$
\begin{aligned}
\boldsymbol{f}_{0}^{\text {har }} & =\frac{1}{T} \int_{0}^{T} f[\boldsymbol{x}(t), \dot{\boldsymbol{x}}(t)] \mathrm{d} t \\
\overline{\boldsymbol{f}}^{\text {har }} & =\frac{2}{T} \int_{0}^{T} \boldsymbol{f}[\boldsymbol{x}(t), \dot{\boldsymbol{x}}(t)] e^{-i \omega t} \mathrm{~d} t
\end{aligned}
$$

where $T=2 \pi / \omega$. In practice, these can be evaluated efficiently from the first two coefficients of the Fast Fourier Transform of $\boldsymbol{f}[\boldsymbol{x}(t), \dot{\boldsymbol{x}}(t)]$. Figure 1 shows an example of the two linearisation approaches.

\section{Linearised aerodynamic forces with aero-elasticity}

The linearisation is now tested by applying it to each of the non-linear functions in the model (Equation 1) in turn, starting with the aerodynamic loads. To begin, the rotor speed, blade pitch angle and induced velocities are all assumed to be known and constant (the "frozen wake" assumption), to focus on solving Equation (1a) for the blade deflections $\boldsymbol{q}$. The harmonic inputs in this case are:

$$
\boldsymbol{x}=\left[\begin{array}{c}
U_{\infty} \\
\boldsymbol{q}
\end{array}\right]
$$

and the function to be linearised is $\mathbf{f}_{\text {aero }}^{k}(\mathbf{x}, \dot{\mathbf{x}})$, the aerodynamic forces on an annulus $k$ (similar to Figure 1). Using Equation (8), Equation (1a) can be written as two linear equations for the constant and harmonic parts:

$$
\begin{array}{r}
\boldsymbol{K} \boldsymbol{q}_{0}=\sum_{k} f_{0}^{k} \\
{\left[-\omega^{2} \boldsymbol{M}+i \omega \boldsymbol{C}+\boldsymbol{K}\right] \overline{\boldsymbol{q}}=\sum_{k} \overline{\boldsymbol{f}}^{k}}
\end{array}
$$

In the tangent linearisation, $f_{0}$ and $\bar{f}$ are given by Equation (11) as:

$$
\begin{aligned}
\boldsymbol{K} \boldsymbol{q}_{0} & =\sum_{k} f^{k}\left(\boldsymbol{x}_{0}, \mathbf{0}\right) \\
{\left[-\omega^{2} \boldsymbol{M}+i \omega \boldsymbol{C}+\boldsymbol{K}-\sum_{k}\left(\boldsymbol{K}_{\boldsymbol{q}}^{k}+i \omega \boldsymbol{C}_{\boldsymbol{q}}^{k}\right)\right] \overline{\boldsymbol{q}} } & =\sum_{k} \boldsymbol{K}_{U}^{k} \bar{U}
\end{aligned}
$$

The forces are evaluated at $\boldsymbol{x}_{0}^{\mathrm{T}}=\left[\begin{array}{ll}U_{0} & \mathbf{0}^{\mathrm{T}}\end{array}\right]$, and the tangent matrices from Equation (9) are partitioned as $K_{f}=\left[\begin{array}{ll}K_{U} & K_{q}\end{array}\right]$ and $C_{f}=\left[\begin{array}{ll}C_{U} & C_{q}\end{array}\right]$ to match the partition of $\boldsymbol{x}$ in Equation (13). This allows the $\boldsymbol{q}$ terms in the tangent matrices to be brought to the left-hand side, accounting for linear aero-elasticity.

In the harmonic linearisation, Equations (14) must be solved together with Equations (12) using an iterative procedure.

\subsection{Method: harmonic, tangent and non-linear calculations}

Results were calculated for a grid of mean wind speeds, harmonic amplitudes and frequencies (Table 1), comparing the harmonic and tangent linearisations to reference non-linear numeric simulations using Bladed [15]. More details are given in Appendix 
Table 1: Mean wind speeds, harmonic amplitudes and frequencies for testing linearisation accuracy. The frequencies were chosen to span roughly the range from floating platform natural frequencies to the 'extreme operating gust' used in wind turbine design [24].

\begin{tabular}{ll}
\hline Mean wind speeds & 8 and $16 \mathrm{~m} \mathrm{~s}^{-1}$ \\
Harmonic amplitudes & $1 \mathrm{~m} \mathrm{~s}^{-1}$ to $5 \mathrm{~m} \mathrm{~s}^{-1}$ \\
Harmonic frequencies & $0.03,0.10,0.32,1.00$ and $3.16 \mathrm{rad} \mathrm{s}^{-1}$ \\
\hline
\end{tabular}

A of the procedure for solving the harmonic linearisation and the numeric simulations, and the full dataset is available in [25]. The turbine parameters are taken from the NREL 5 MW turbine [20].

The results are compared using the peak-peak error, which is a simple measure which is relevant whether fatigue or extreme loads are of interest.

\subsection{Results}

One set of solutions, for an amplitude of $5 \mathrm{~m} \mathrm{~s}^{-1}$ about the mean $8 \mathrm{~m} \mathrm{~s}^{-1}$, is shown in Figure 4; further results are given in Appendix B.

Figure 5 show the distributed blade loads at several points along the blade, which together make up the rotor thrust and torque shown in Figure 4. Most of the non-linear behaviour due to stalling can be seen in the midspan of the blade. The larger loops towards the tip are due to the greater blade deflections there.

Figures 6-7 show the error between the linearised results and the non-linear simulations (numeric data given in Appendix B), shown as the peak-peak error normalised by the Bladed peak-peak value. For small wind speed perturbations, both harmonic and tangent linearisations give small errors. The errors of both increase as the size of the wind speed perturbations increases, but the increase is greater for the tangent linearisation. At a mean wind speed of $8 \mathrm{~m} \mathrm{~s}^{-1}$, which is the case shown in Figures 4-5, the harmonic linearisation is up to 4 times better than the tangent linearisation. At the higher mean wind speed of $16 \mathrm{~m} \mathrm{~s}^{-1}$, the behaviour is more linear. The improvements are less significant, but the error in the rotor torque is still reduced by a factor of 2 .

Overall, the maximum error in the harmonic linearisation results is $8.4 \%$ of the peakpeak range, and occurs in the rotor torque. If the wind speed variations are below $3 \mathrm{~m} \mathrm{~s}^{-1}$, the maximum error is $3.4 \%$. The maximum error in the rotor thrust is $5.7 \%$ in the harmonic linearisation and $24.1 \%$ in the tangent linearisation.

\section{Harmonic linearisation of wake dynamics}

In the previous section the wake dynamics were neglected and the aero-elastic response to wind speed variations was examined; now the wake dynamics are linearised while neglecting the blade dynamics. Because the aerodynamic calculations in each annulus are independent, only one annulus need be considered at a time. Again, the rotor speed and blade pitch angle are assumed constant, giving a vector of harmonic inputs

$$
\boldsymbol{x}=\left[\begin{array}{c}
U_{\infty} \\
u
\end{array}\right]
$$

where $u$ is the induced axial velocity in the annulus. The function to be linearised is $\dot{u}=g(\mathbf{x})$ from Equation (1c). In this case, a harmonic steady-state solution is sought for the induced velocity $u$, which must have a constant mean value. These requirements are 

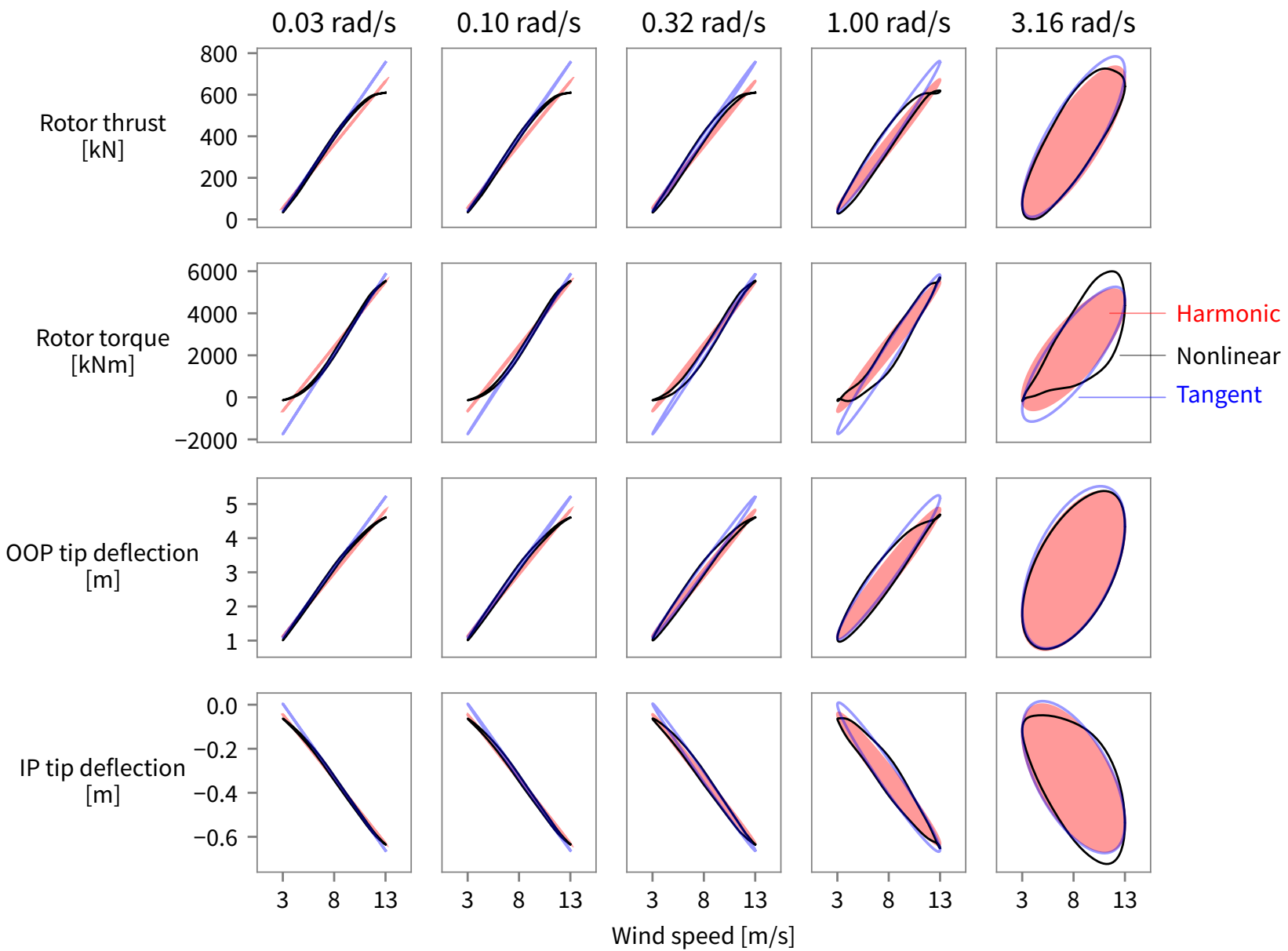

Figure 4: Example linearisation results for rotor loads and blade tip in-plane (IP) and out-of-plane (OOP) deflections, plotted against the harmonic wind speed input $\left(8 \pm 5 \mathrm{~m} \mathrm{~s}^{-1}\right)$. The columns correspond to wind speed variations of different frequencies. The aerodynamic thrust and torque is noticeably non-linear, due to the aerofoils stalling during part of the large cyclic variation in wind speed. The deflection responses are also therefore somewhat non-sinusoidal. The harmonic linearisation represents the behaviour reasonably well; the tangent linearisation tends to overestimate the peak thrust. 


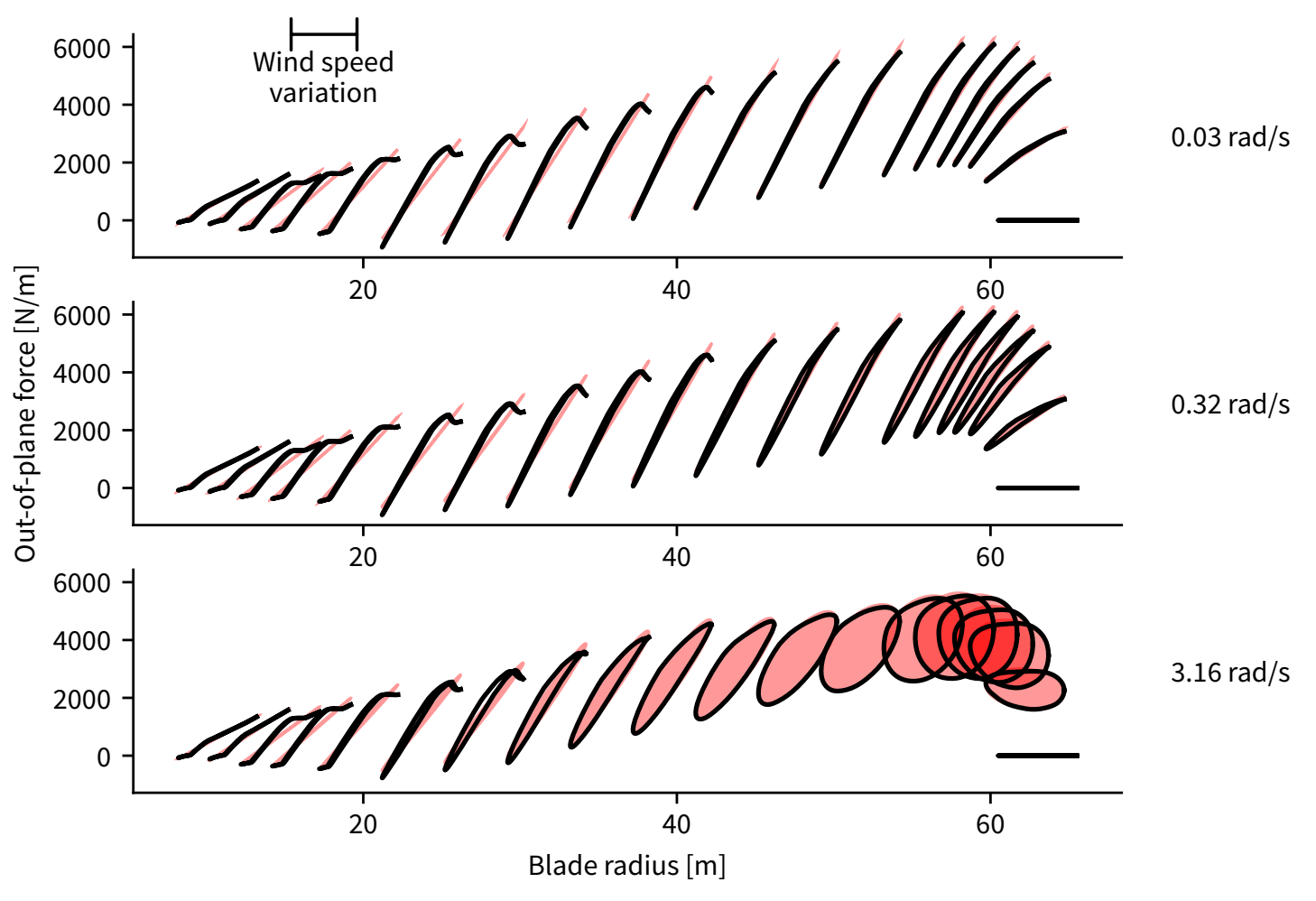

Figure 5: Out-of-plane aerodynamic blade loading at several points along the blade, for three frequencies.

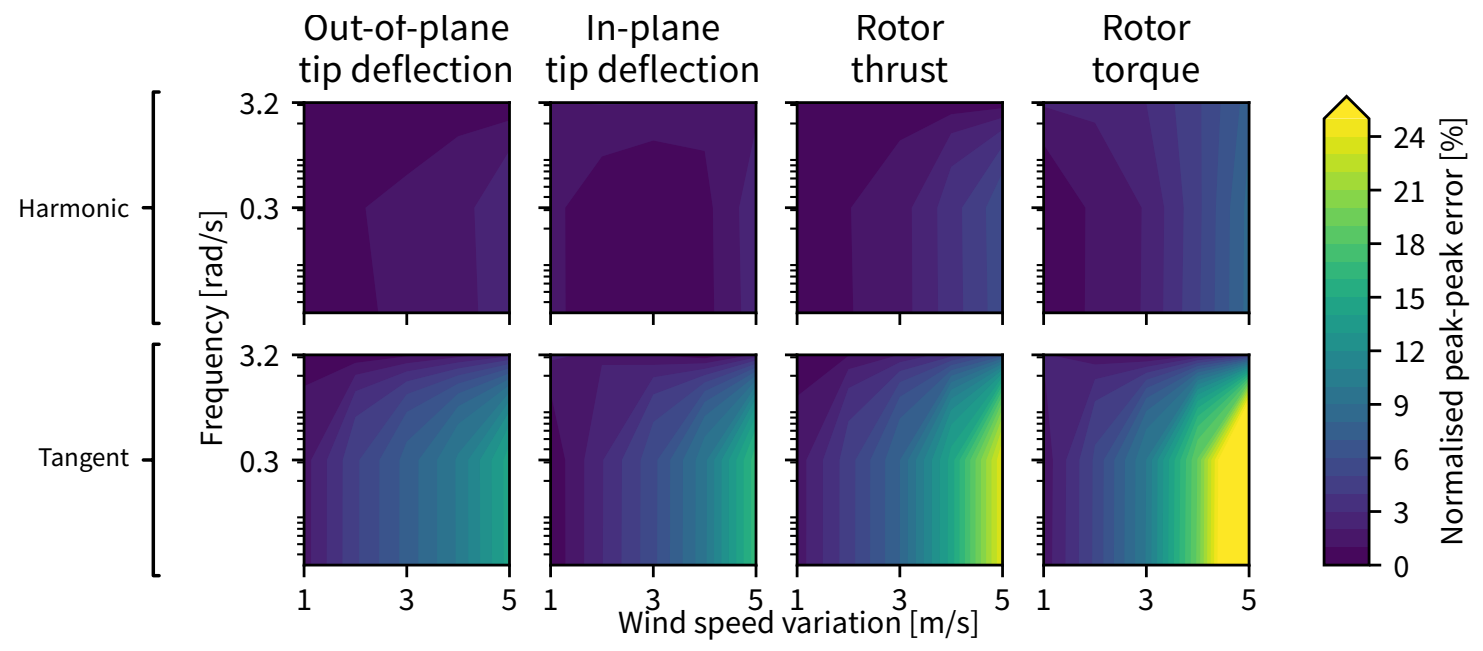

Figure 6: Error between linearised and non-linear results, for mean wind speed of $8 \mathrm{~m} \mathrm{~s}^{-1}$ (below rated). The error is defined as the peak-peak range of the linearised result, normalised by the peak-peak range of the nonlinear result. 
satisfied by solving the non-linear equations

$$
\begin{aligned}
g_{0} & =0 \\
\bar{g} & =i \omega \bar{u}
\end{aligned}
$$

with $g_{0}$ and $\bar{g}$ being calculated numerically according to Equations (12).

As an example, Figure 8 shows results for harmonic wind speed inputs at low, medium and high frequencies. At low frequencies the response is quasi-static, following the contour line for $\dot{u}=0$. Since the quasi-static response is non-linear in wind speed, the linearisation deviates from the non-linear solution. At higher frequencies, the wake dynamics cause a lag between the change in wind speed and the change in induced velocity, and the non-linearity in the quasi-static solution is less important.

\section{Harmonic linearisation of torque and pitch control}

The results in the previous sections were calculated for constant rotational speed of the rotor, and a constant blade pitch angle. In reality, both of these are actively controlled by the wind turbine controller, so the control system is now reintroduced into the model in two steps: firstly the generator torque function $Q_{g}$ is linearised in isolation, assuming the rotor speed is known (involving Equation 1d). Secondly, the linearised aerodynamic and generator torques are used to solve for the rotor speed response using Equations (1b), (1d) and (1e).

\subsection{Harmonic linearisation of generator torque}

The function to be linearised is now the generator torque $Q_{g}\left(\Omega_{g}\right)$, shown in Figure 2 . The controller acts on the low-pass filtered generator speed (Equation 1d), so first the harmonic filtered generator speed response must be found, then the function $Q_{g}$ must be linearised.

Putting a harmonic rotor speed, as described by Equation (7), into Equation (1d) gives the mean value and complex amplitude of the filtered speed as

$$
\begin{aligned}
\left(\Omega_{g}\right)_{0} & =G \Omega_{0} \\
\overline{\Omega_{g}} & =\frac{G \bar{\Omega}}{1+i \omega / \omega_{c}}
\end{aligned}
$$

where $\omega$ is the frequency of the harmonic signals, and $\omega_{c}$ is the filter corner frequency.

The harmonic linearisation of $Q_{g}$ is found numerically by applying Equation (12) to the simulated response of the torque controller over a cycle.

The accuracy of the linearisation depends on the mean value and the amplitude of the rotor speed, and hence which region of the torque curve is involved. Figure 9 compares the non-linear and linearised generator torques corresponding to various choices of the harmonic wind speed. Results are shown for harmonic variations in rotor speed at three frequencies. Because of the filtering of the generator speed signal, at high frequencies the torque variations are relatively small and the linearisation performs well. At lower frequencies the non-linearity is more pronounced, especially around the rated generator speed, but the linearisation still gives a fairly good representation of the generator torque.

\subsection{Harmonic solution for rotor speed}

Since in a variable-speed turbine the rotor speed is not known in advance, the torque controller linearisation from the previous section is now combined with the rotor rotational dynamics (Equation 1b) using the linearised aerodynamic loads derived in Section 4 to solve for the rotor speed. 


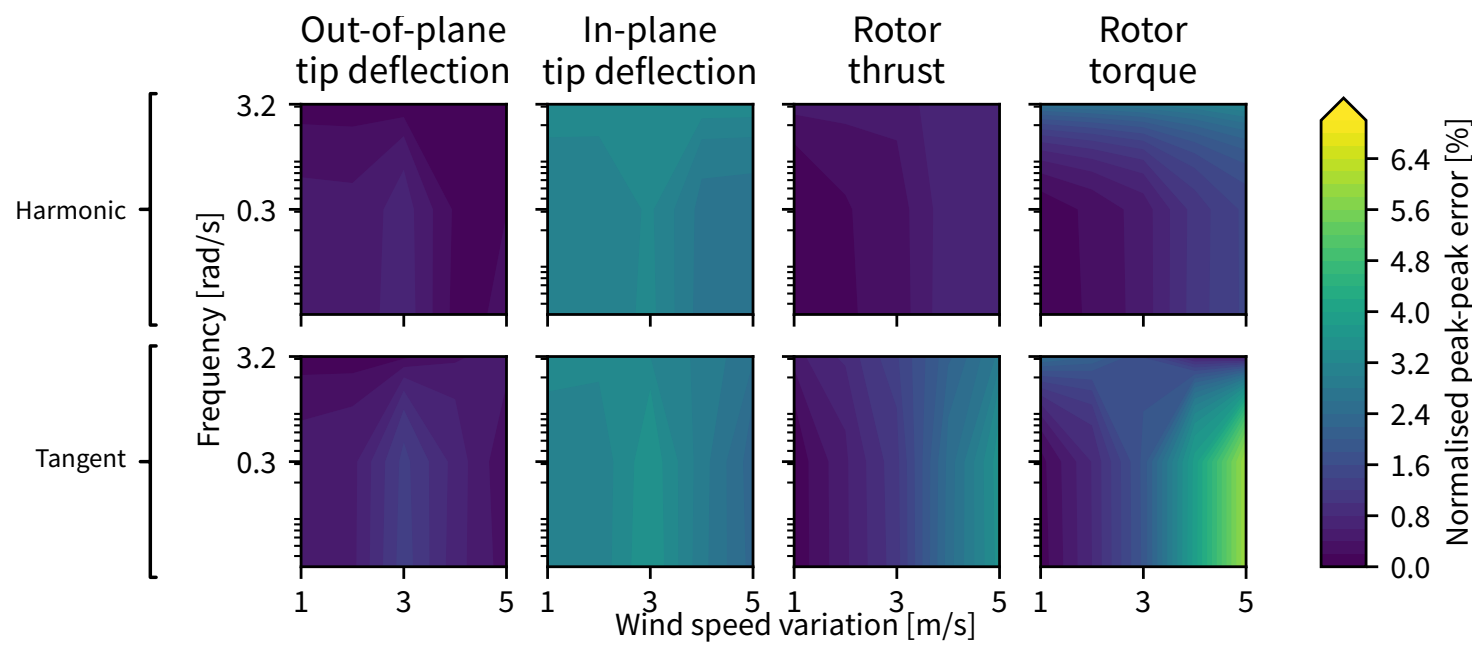

Figure 7: As in Figure 6, for mean wind speed of $16 \mathrm{~m} \mathrm{~s}^{-1}$ (above rated).

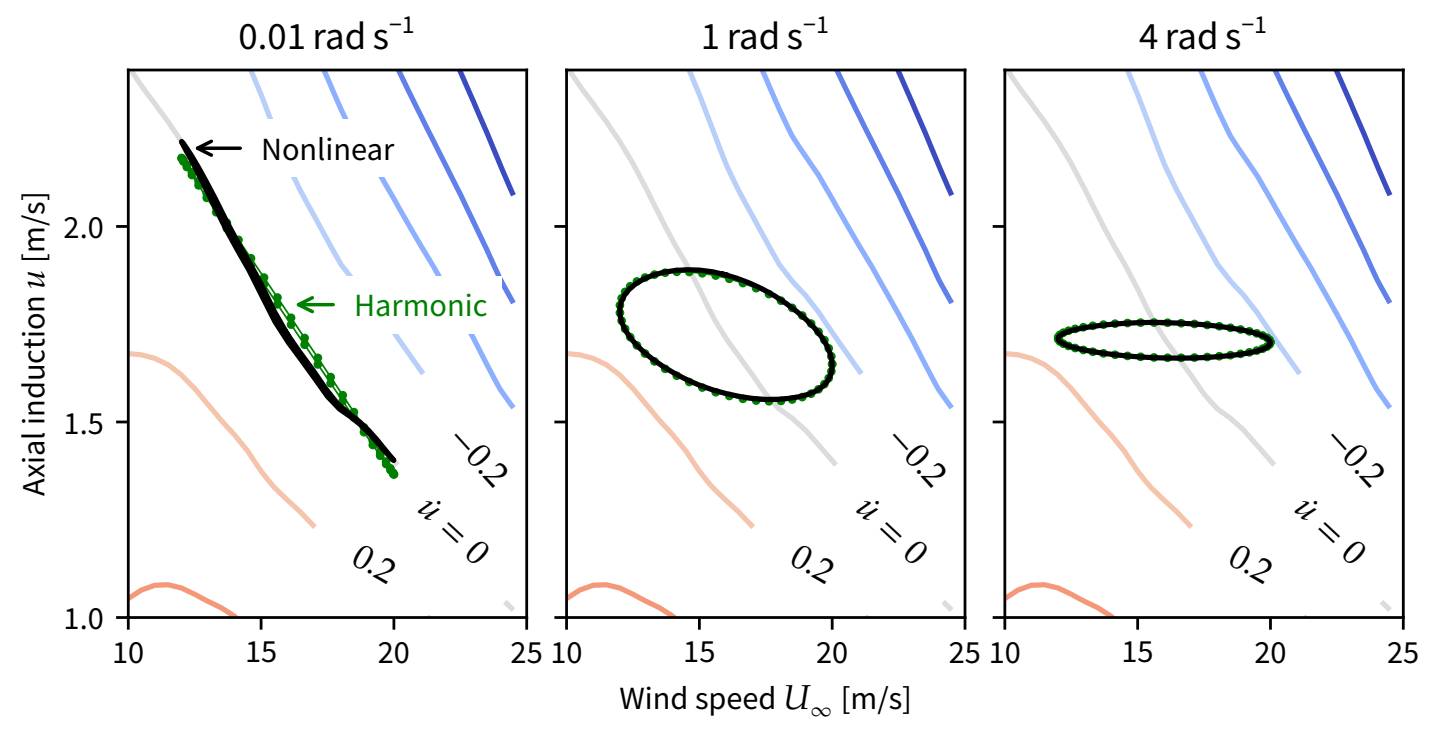

Figure 8: Wake solution for harmonic wind input of $(16 \pm 4) \mathrm{m} \mathrm{s}^{-1}$ at three frequencies. 

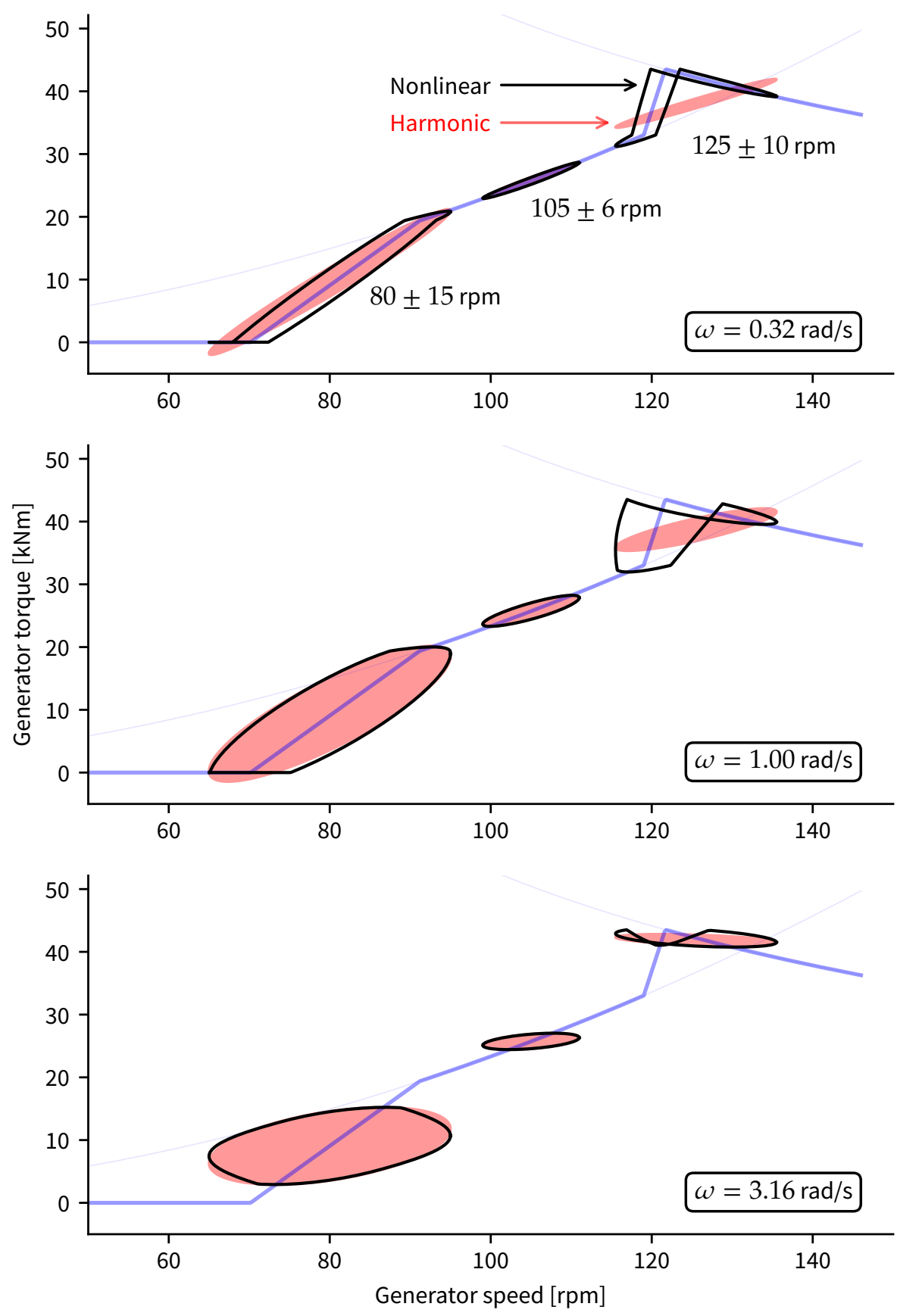

Figure 9: Examples of linearised harmonic generator torque compared to the underlying non-linear behaviour, for three frequencies. The lines in the background are the same as Figure 2. The example at the left is mildly nonlinear at the corners of the torque curve. For the example in the middle the underlying behaviour is quadratic, but over the range shown it is well approximated by the linear solution. The example at the right is highly non-linear at low frequencies. At high frequencies the filtering of the generator speed measurement reduces the torque variation, improving the accuracy of the harmonic linearisation. 
Table 2: Mean wind speeds, harmonic amplitudes and frequencies for testing linearisation accuracy of the control system and rotor response. The frequencies are the same as in Table 1, but a finer grid of mean wind speeds has been tested.

\begin{tabular}{ll}
\hline Mean wind speeds & $6 \mathrm{~m} \mathrm{~s}^{-1}$ to $15 \mathrm{~m} \mathrm{~s}^{-1}$ \\
Harmonic amplitudes & $1 \mathrm{~m} \mathrm{~s}^{-1}$ to $3 \mathrm{~m} \mathrm{~s}^{-1}$ \\
Harmonic frequencies & $0.03,0.10,0.32,1.00$ and $3.16 \mathrm{rad} \mathrm{s}^{-1}$ \\
\hline
\end{tabular}

The harmonic linearisation of Equation (1b) give two linear equations governing the harmonic rotor response:

$$
\begin{aligned}
\left(Q_{a}\right)_{0}-G\left(Q_{g}\right)_{0} & =0 \\
\overline{Q_{a}}-G \overline{Q_{g}} & =i \omega J \bar{\Omega}
\end{aligned}
$$

The aerodynamic torque and generator torque are both non-linear functions of the rotor speed, as defined by Equation (2) and Equation (4) respectively, so Equations (19) must be solved numerically.

Below the rated wind speed of the turbine, this is enough, but at higher wind speeds the torque controller switches to constant-power mode and the pitch controller becomes active. This adds an additional state, the pitch controller integral error $I_{\epsilon}$ (Equation 1e). The harmonic solution for $I_{\epsilon}$ is:

$$
\begin{aligned}
0 & =\left(\Omega_{g}\right)_{0}-\Omega_{\text {rated }} \\
\overline{I_{\epsilon}} & =\frac{\overline{\Omega_{g}}}{i \omega}
\end{aligned}
$$

where $\left(\Omega_{g}\right)_{0}$ and $\overline{\Omega_{g}}$ are the filtered generator speed given by Equation (18).

The non-zero pitch angle must now be taken into account when calculating the aerodynamic torque $Q_{a}$. The pitch angle is calculated from the speed error and integral error as shown by Equation (5), which in the harmonic case becomes

$$
\begin{aligned}
\theta_{0} & =G_{K} K_{I}\left(I_{\epsilon}\right)_{0} \\
\bar{\theta} & =G_{K}\left[K_{P}+\frac{K_{I}}{i \omega}\right] \overline{\Omega_{g}}
\end{aligned}
$$

The gain schedule factor $G_{K}$ is calculated at the mean pitch angle, on the assumption that it varies slowly relative to the amplitude of the pitch variations. By substituting Equation (21a) into (6), $G_{K}$ may be found as a function of $I_{\epsilon}$ as

$$
G_{K}=\frac{-1+\sqrt{1+4 a}}{2 a} \quad \text { where } a=K_{I} I_{\epsilon} / \theta_{2}
$$

Given a known harmonic wind speed input, the harmonic rotor speed and pitch angle are found as before using a root-finding algorithm to solve Equations (19) and (20) for $\left(\Omega_{g}\right)_{0}, \overline{\Omega_{g}},\left(I_{\epsilon}\right)_{0}$ and $\overline{I_{\epsilon}}$.

This linearisation does not account for the limits on pitch angle and rate which are implemented in the non-linear controller. In normal operation the pitch rate limits should not be reached, so this should be acceptable for a first approach to linearising the controller behaviour. The lack of pitch angle end-stops does lead to errors in the following results.

\subsection{Method: comparison of harmonic and non-linear solutions}

The wind turbine response has been solved for harmonic wind speed input over a grid of conditions (Table 2), and the harmonic linearisation compared to reference non-linear 

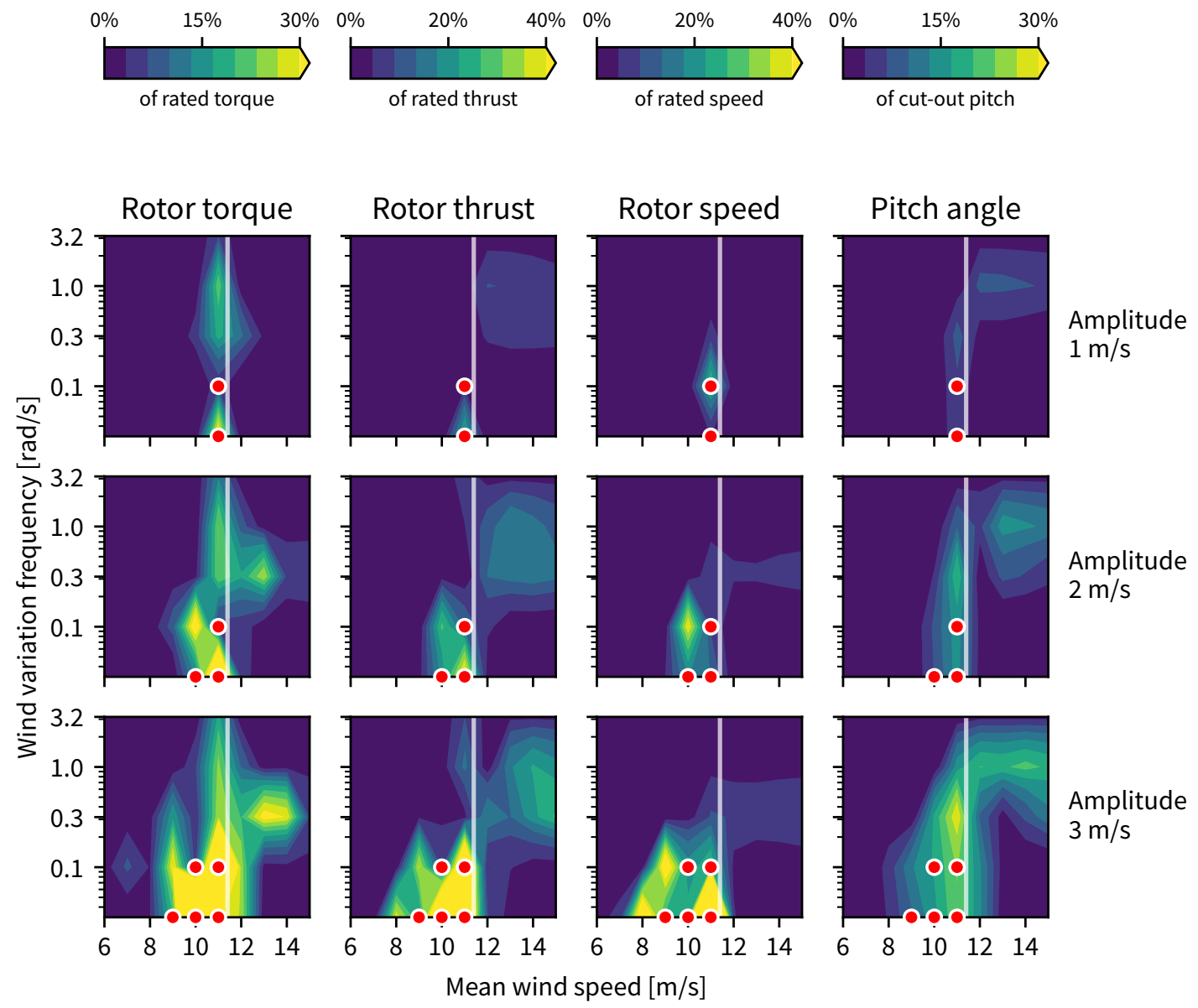

Figure 10: Error in peak-peak value of linearised results compared to non-linear simulations. In some areas it was not possible to find a harmonic solution; these are shown by the red dots. The colour scale for each column is different, as shown by the scales at the top. In each column the error is normalised by a representative value: the rated rotor torque $(4.18 \mathrm{MN} \mathrm{m})$, the rated rotor thrust $(721 \mathrm{kN})$, the rated rotor speed $(12.1 \mathrm{rpm})$ and the maximum operation blade pitch angle $\left(23.2^{\circ}\right)$.

simulations. These reference results were simulated in the time domain using a nonlinear implementation of the controller as described and verified in Lupton [22], and the full results dataset is available in [25].

In the harmonic solution, since the number of equations to be solved depends on whether the pitch controller is active, for simplicity the pitch controller is assumed to be active only when the mean wind speed is above the nominal rated wind speed of $11.4 \mathrm{~m} \mathrm{~s}^{-1}$. This will cause inaccuracies when the mean wind speed is below rated but part of the harmonic variation rises above rated; this is visible in the following results.

\subsection{Results}

Figure 10 shows the error in the peak-peak value of the linearised results compared to the non-linear simulations. The error has been normalised by a representative value of each variable: respectively, the rated rotor torque, rated rotor thrust, rated rotor speed and blade pitch angle at cutout. Generally, the errors are fairly small for small variations in wind speed, but increase for larger variations in wind speed. 
In some cases it has not been possible to find a correct harmonic steady-state solution for the rotor speed and pitch angle. These cases are shown by red dots in Figure 10. This occurs when the wind speed crosses the rated wind speed of the turbine, meaning that the pitch controller should be active for part of the cycle, but in these results the pitch controller is included only for mean wind speeds above the rated wind speed $\left(11.4 \mathrm{~m} \mathrm{~s}^{-1}\right)$. Therefore the correct solution for cases just below the rated wind speed has not been found.

Figure 11 gives more detail by plotting the variation with time of the non-linear and linearised responses at a frequency of $0.32 \mathrm{rad} \mathrm{s}^{-1}$. Additional results at different frequencies are in Appendix C.

The linearised results show the best agreement with the non-linear simulations at mean wind speeds far from the turbine's rated wind speed (left and right columns). When the mean wind speed is close to rated (middle columns), the generator torque behaviour is much more non-linear, as shown on the right-hand side of Figure 2. The pitch angle limits also take effect close to rated, but are not captured by the present linearisation: just below rated, the blades begin to pitch for part of the cycle, and just above rated, the blades may hit the minimum-pitch limit for part of the cycle.

\section{Implications for linearised modelling of wind turbines}

While the details of the results are specific to the particular NREL 5MW blade used in these calculations, we expect the overall performance of the linearisation methods should be similar in most comparable large, variable speed, variable pitch wind turbines. In fact there are two reasons why real-world performance of the linearisation might be expected to be better than shown here.

We have assumed a uniform wind speed applied across the whole rotor, but this is the worst case for rotor loading. Wind speed variations due to rigid body motion of a floating platform may come close to this worst case, but variations in wind speed due to turbulence have less spatial correlation, which would reduce the level of non-linearity in the overall rotor loading and improve the accuracy of the linearisations.

While the controller used here is representative, it is unlikely to be as well designed as a real wind turbine controller. The same discontinuities in the control system which cause difficulties for the linearisation are also demanding for the wind turbine drive train, pitch system and blade loading. A real controller design will consider these issues, so if anything smoother behaviour more amenable to linearisation should be expected.

There are two main limitations to the linearised control system model implemented in this paper, which could be addressed to improve the accuracy of the linearisation:

1. The pitch controller is only active when the mean wind speed is above the rated wind speed of the turbine. This means the behaviour is poor when the wind speed passes from below to above rated transiently, since the non-linear controller starts to pitch the blades but the linearised controller cannot. Improving this should address the issues visible in Figure 10 where solutions could not be found for wind speeds below rated.

2. The non-linearities in the pitch control system were not fully captured, because the harmonic pitch angle was solved based on the theoretical PID controller equation rather than the actual non-linear control output. Calculating the pitch angle numerically in a similar way to the calculation of the linearised generator torque may give better results around rated when pitch angle limits are reached. 

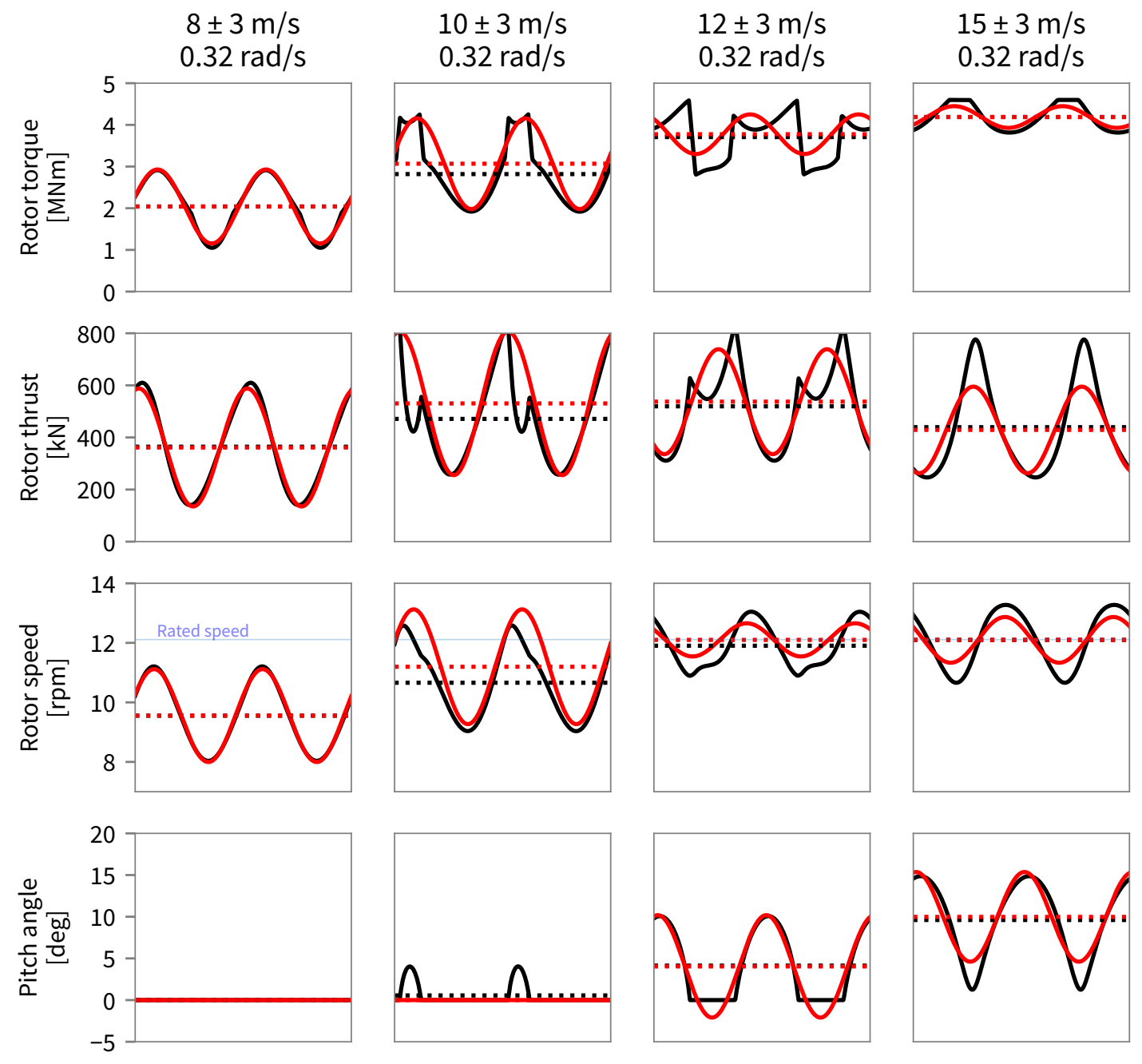

Figure 11: Comparison of linearised and non-linear results for harmonic wind speed input varying at $0.32 \mathrm{rad} \mathrm{s}^{-1}$. Dotted lines show mean values. 
This paper focused on harmonic inputs, but in some cases stochastic turbulence inputs are more appropriate. The extension of this method to 'stochastic linearisation' should be straightforward: instead of minimising the mean squared error over one cycle of the harmonic input, instead the expectation of the squared error is minimised [see for example 26, chapter 6]. Allowing for simultaneous random and deterministic inputs is possible although more complicated [26, chapter 7].

\section{Conclusions}

This paper has demonstrated how harmonic linearisation can be used to improve approximations of the non-linear aerodynamic loads, wake dynamics, and control system behaviour of a wind turbine, by comparing the results to tangent linearisation and nonlinear reference simulations of the NREL 5MW turbine. This is the first time harmonic linearisation has been applied to wind turbine aerodynamics and control system behaviour. In summary the results are:

- Harmonic linearisation reduced the error in approximating the aerodynamic loads by 2-4 times compared to tangent linearisation, with a maximum absolute error of $8.4 \%$ over the range of conditions tested.

- The method can be extended to include a linearisation of the wake dynamics, avoiding the need for a "frozen wake" assumption.

- The control system presents a bigger challenge, especially in the area around rated wind speed when the controller behaviour is less linear and the two controllers interact. Poor results are obtained under these conditions (rotor torque and thrust peak-peak errors of up to $40 \%$ or rated torque and thrust), but elsewhere non-linear behaviour is well approximated (errors below 10\%).

These results show that harmonic linearisation is a promising approach to improve the accuracy of linearised models of wind turbine response, enabling the use of fast frequencydomain modelling methods for initial design and optimisation of wind turbines. Further work has been identified to improve the linearisation of the control system behaviour and to extend the approach to stochastic inputs.

In this paper, the structural dynamics, wake dynamics and control system dynamics have been considered separately. In a forthcoming paper we will present a more complete example of a linearised frequency-domain model of a floating wind turbine, in which the platform and structural dynamic response to non-linear aerodynamic forces are solved together, both with and without the control system behaviour.

\section{Acknowledgements}

This work was funded by an EPSRC doctoral training award (ref. 1089390) and supported by GL Garrad Hassan.

\section{References}

[1] R. C. Lupton, R. S. Langley, Complex but negligible: Non-linearity of the inertial coupling between the platform and blades of floating wind turbines, Renewable Energy 134 (2019) 710-726. doi:10.1016/j.renene.2018.11.036. 
[2] R. C. Lupton, R. S. Langley, Scaling of slow-drift motion with platform size and its importance for floating wind turbines, Renewable Energy 101 (2017) 1013-1020. doi:10.1016/j.renene.2016.09.052.

[3] A. Halfpenny, Dynamic Analysis of Both On and Offshore Wind Turbines in the Frequency Domain, Ph.D. thesis, University College London, 1998.

[4] K. O. Merz, M. Muskulus, G. Moe, A simple frequency-domain method for stress analysis of stall-regulated wind turbines: Frequency-domain analysis of stall-regulated turbines, Wind Energy 15 (2012) 773-798. doi:10.1002/we.504.

[5] T. G. van Engelen, Frequency Domain Analysis of Offshore Wind Turbines, Technical Report, ECN, 2004.

[6] P. van der Male, K. N. van Dalen, A. V. Metrikine, The effect of the nonlinear velocity and history dependencies of the aerodynamic force on the dynamic response of a rotating wind turbine blade, Journal of Sound and Vibration 383 (2016) 191-209. doi:10.1016/j.jsv.2016.07.031.

[7] F. E. Durá, F. M. Gimenez, J. S. Corretge, Fast Estimation of the Damage Equivalent Load in Blade Geometry Multidisciplinar Optimization, Journal of Solar Energy Engineering 139 (2017) 041008. doi:10.1115/1.4036636.

[8] M. Hall, B. Buckham, C. Crawford, Evolving offshore wind: A genetic algorithmbased support structure optimization framework for floating wind turbines, IEEE, 2013, pp. 1-10. doi:10.1109/OCEANS-Bergen.2013.6608173.

[9] J. M. Kluger, T. P. Sapsis, A. H. Slocum, A reduced-order, statistical linearization approach for estimating nonlinear floating wind turbine response statistics, in: The 26th International Ocean and Polar Engineering Conference, International Society of Offshore and Polar Engineers, 2016.

[10] T. Burton, D. Sharpe, N. Jenkins, E. Bossanyi, Wind Energy Handbook, John Wiley \& Sons, 2011.

[11] A. Sabale, K. V. N. Gopal, Nonlinear aeroelastic response of wind turbines using Simo-Vu-Quoc rods, Applied Mathematical Modelling 65 (2019) 696-716. doi:10. 1016/j.apm.2018.09.003.

[12] M. I. Kvittem, T. Moan, Frequency Versus Time Domain Fatigue Analysis of a Semisubmersible Wind Turbine Tower, Journal of Offshore Mechanics and Arctic Engineering 137 (2014) 011901. doi:10.1115/1.4028340.

[13] M. Philippe, A. Babarit, P. Ferrant, Comparison of Time and Frequency Domain Simulations of an Offshore Floating Wind Turbine, in: ASME 2011 30th International Conference on Ocean, Offshore and Arctic Engineering, 2011, pp. 589-598. doi:10.1115/OMAE2011-49722.

[14] J. M. Jonkman, B. J. Jonkman, FAST modularization framework for wind turbine simulation: Full-system linearization, Journal of Physics: Conference Series 753 (2016) 082010. doi:10.1088/1742-6596/753/8/082010.

[15] Garrad Hassan, Bladed Theory Manual, Technical Report, 2011.

[16] K. H. Lee, Responses of Floating Wind Turbines to Wind and Wave Excitation, Ph.D. thesis, MIT, 2005. 
[17] J. Olondriz, J. Jugo, I. Elorza, S. Alonso-Quesada, A. Pujana-Arrese, Alternative linearisation methodology for aero-elastic Floating Offshore Wind Turbine non-linear models, Journal of Physics: Conference Series 1037 (2018) 062019. doi:10.1088/1742-6596/1037/6/062019.

[18] M. Vidyasagar, Nonlinear systems analysis, 2nd ed ed., Prentice Hall, 1993.

[19] R. S. Langley, The linearisation of three dimensional drag force in random seas with current, Applied Ocean Research 6 (1984) 126-131.

[20] J. Jonkman, S. Butterfield, W. Musial, G. Scott, Definition of a 5-MW Reference Wind Turbine for Offshore System Development, Technical Report February, National Renewable Energy Laboratory, Golden, USA, 2009.

[21] J. B. de Vaal, M. O. L. Hansen, T. Moan, Effect of wind turbine surge motion on rotor thrust and induced velocity, Wind Energy 17 (2014) 105-121. URL: http: //doi.wiley.com/10.1002/we.1562. doi:10.1002/we.1562.

[22] R. C. Lupton, Frequency-Domain Modelling of Floating Wind Turbines, Ph.D. thesis, University of Cambridge, 2015. URL: https ://doi .org/10.17863/CAM.14119.

[23] R. S. Langley, Harmonic linearisation of geometrically non-linear finite element models, Computers \& Structures 28 (1988) 165-172. URL: http://linkinghub.elsevier.com/retrieve/pii/0045794988900363. doi:10.1016/0045-7949(88)90036-3.

[24] International Electrotechnical Commission, Wind turbines - Part 1: Design requirements (edition 3), 2005.

[25] R. C. Lupton, ricklupton/wind-turbine-harmonic-linearisation-paper, 2018. doi:10 . $5281 /$ zenodo. 1484513.

[26] J. B. Roberts, P. D. Spanos, Random vibration and statistical linearization, John Wiley \& Sons, 1990.

[27] E. Jones, T. Oliphant, P. Peterson, Others, SciPy: Open source scientific tools for Python, 2001. URL: http://www. scipy . org/. 


\section{Appendix A. Harmonic linearisation method and non-linear simulations}

Appendix A.1. Harmonic linearisation

The solution procedure for the harmonic linearisation is broadly as follows:

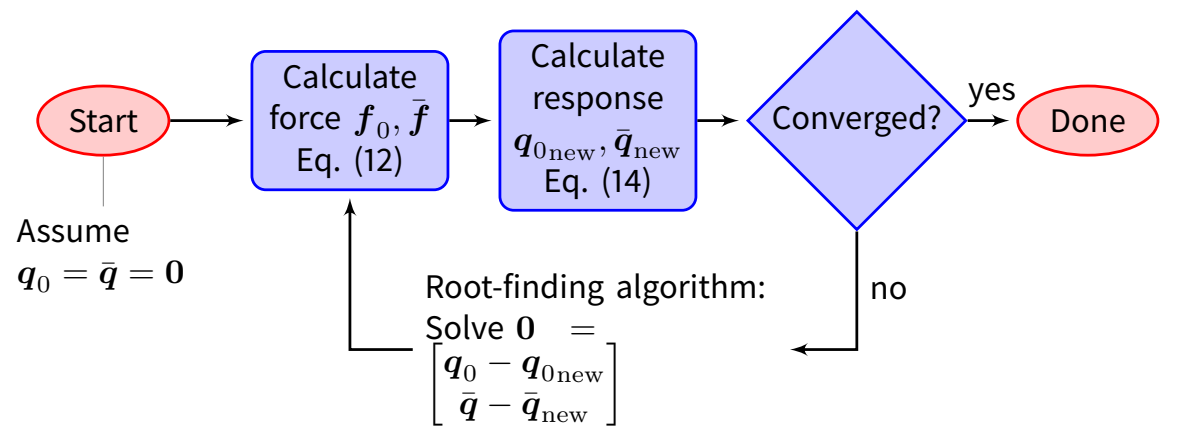

where the iteration is controlled by a numerical multi-dimensional root-finding algorithm. In the first case, finding the structural response, there is little difficulty in finding the solution since the structural response is linear, but in the later cases and in general an iterative numerical solution is needed. The 'hybr' method implemented in SciPy [27] was used.

Appendix A.2. Non-linear reference results

The non-linear reference results were found from numerical simulation of a Blade Element Momentum (BEM) aerodynamic model combined with a flexible multi-body dynamics code described and validated in Lupton [22].

Simulations were run for the conditions listed in Table 1 for the minimum of $60 \mathrm{~s}$ or 5 cycles, leading to the following parameters:

$$
\begin{array}{lll}
0.03 \mathrm{rad} \mathrm{s}^{-1} & t_{\max }=1987 \mathrm{~s} & \Delta t=1 \mathrm{~s} \\
0.10 \mathrm{rad} \mathrm{s}^{-1} & t_{\max }=628 \mathrm{~s} & \Delta t=1 \mathrm{~s} \\
0.32 \mathrm{rad} \mathrm{s}^{-1} & t_{\max }=199 \mathrm{~s} & \Delta t=0.1 \mathrm{~s} \\
1.00 \mathrm{rad} \mathrm{s}^{-1} & t_{\max }=63 \mathrm{~s} & \Delta t=0.1 \mathrm{~s} \\
3.16 \mathrm{rad} \mathrm{s}^{-1} & t_{\max }=60 \mathrm{~s} & \Delta t=0.01 \mathrm{~s}
\end{array}
$$

The first parts of the simulations are discarded, removing initial transients, and only the final cycle is used.

\section{Appendix B. Additional aerodynamic force results}

Figure B.12 shows a different view of the results in Figure 4 by plotting against time. Table B.3 gives numeric data shown in Figures 6-7.

\section{Appendix C. Additional control linearisation results}

Figures C.13-C.14 show similar results to those shown in Figure 11, for a lower and a higher frequency respectively. 

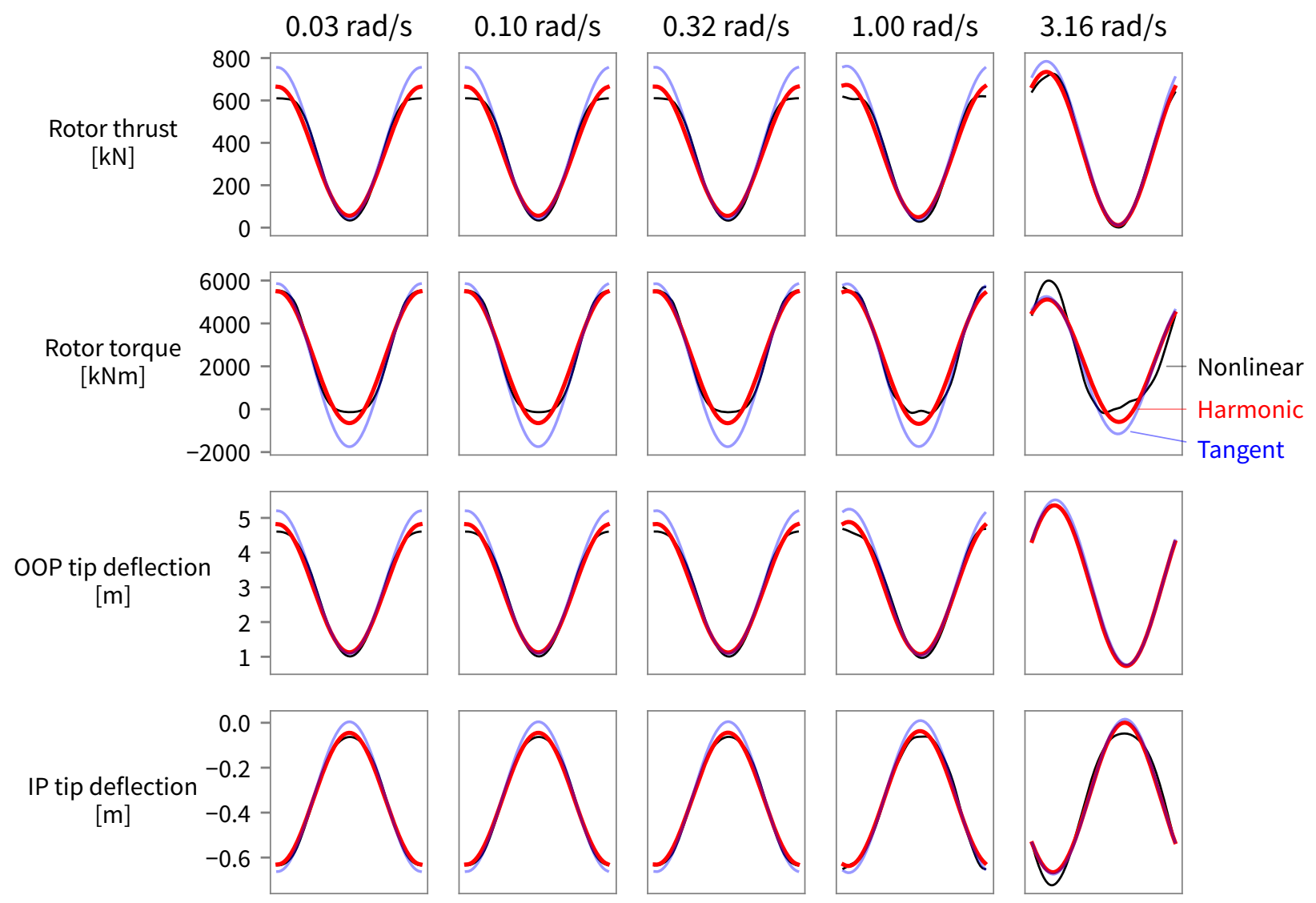

Figure B.12: Example linearisation results for rotor loads and blade tip in-plane (IP) and out-of-plane (OOP) deflections, plotted over one cycle of harmonic wind speed input $\left(8 \pm 5 \mathrm{~m} \mathrm{~s}^{-1}\right)$. The columns correspond to wind speed variations of different frequencies. 
Table B.3: Error of linearisations, as shown in Figures 6-7. The maximum error for various amplitudes of wind speed variation $A$ is shown.

\begin{tabular}{lrrrr}
\hline & $\begin{array}{r}\text { Out-of-plane } \\
\text { defl. }\end{array}$ & $\begin{array}{r}\text { In-plane } \\
\text { defl. }\end{array}$ & $\begin{array}{r}\text { Rotor } \\
\text { thrust }\end{array}$ & $\begin{array}{r}\text { Rotor } \\
\text { torque }\end{array}$ \\
\hline Mean wind speed $8 \mathrm{~m} \mathrm{~s}^{-1}$ & & & \\
Harmonic: & & & & \\
$A<1 \mathrm{~m} \mathrm{~s}^{-1}$ & $0.5 \%$ & $2.0 \%$ & $0.5 \%$ & $2.2 \%$ \\
$A<2 \mathrm{~m} \mathrm{~s}^{-1}$ & $1.0 \%$ & $2.1 \%$ & $1.0 \%$ & $2.6 \%$ \\
$A<3 \mathrm{~m} \mathrm{~s}^{-1}$ & $1.1 \%$ & $2.1 \%$ & $1.5 \%$ & $3.0 \%$ \\
$A<4 \mathrm{~m} \mathrm{~s}^{-1}$ & $1.6 \%$ & $2.1 \%$ & $3.5 \%$ & $4.8 \%$ \\
$A<5 \mathrm{~m} \mathrm{~s}^{-1}$ & $2.8 \%$ & $2.6 \%$ & $5.7 \%$ & $8.4 \%$ \\
Tangent: & & & & \\
$A<1 \mathrm{~m} \mathrm{~s}^{-1}$ & $1.6 \%$ & $2.3 \%$ & $1.4 \%$ & $2.1 \%$ \\
$A<2 \mathrm{~m} \mathrm{~s}^{-1}$ & $4.8 \%$ & $3.0 \%$ & $4.5 \%$ & $5.2 \%$ \\
$A<3 \mathrm{~m} \mathrm{~s}^{-1}$ & $7.4 \%$ & $5.7 \%$ & $8.0 \%$ & $10.1 \%$ \\
$A<4 \mathrm{~m} \mathrm{~s}^{-1}$ & $10.1 \%$ & $9.7 \%$ & $14.4 \%$ & $19.3 \%$ \\
$A<5 \mathrm{~m} \mathrm{~s}^{-1}$ & $14.5 \%$ & $16.7 \%$ & $24.1 \%$ & $34.1 \%$ \\
& & & & \\
\hline
\end{tabular}

Mean wind speed $16 \mathrm{~m} \mathrm{~s}^{-1}$

Harmonic:

\begin{tabular}{lllll}
$A<1 \mathrm{~m} \mathrm{~s}^{-1}$ & $0.5 \%$ & $3.4 \%$ & $0.5 \%$ & $2.6 \%$ \\
$A<2 \mathrm{~m} \mathrm{~s}^{-1}$ & $0.5 \%$ & $3.4 \%$ & $0.5 \%$ & $2.7 \%$ \\
$A<3 \mathrm{~m} \mathrm{~s}^{-1}$ & $0.7 \%$ & $3.4 \%$ & $0.6 \%$ & $2.8 \%$ \\
$A<4 \mathrm{~m} \mathrm{~s}^{-1}$ & $0.7 \%$ & $3.4 \%$ & $0.7 \%$ & $3.0 \%$ \\
$A<5 \mathrm{~m} \mathrm{~s}^{-1}$ & $0.7 \%$ & $3.4 \%$ & $0.8 \%$ & $3.3 \%$ \\
& & & & \\
Tangent: & & & & \\
$A<1 \mathrm{~m} \mathrm{~s}^{-1}$ & $0.5 \%$ & $3.4 \%$ & $0.5 \%$ & $2.5 \%$ \\
$A<2 \mathrm{~m} \mathrm{~s}^{-1}$ & $0.5 \%$ & $3.4 \%$ & $0.9 \%$ & $2.5 \%$ \\
$A<3 \mathrm{~m} \mathrm{~s}^{-1}$ & $1.3 \%$ & $3.6 \%$ & $1.4 \%$ & $2.5 \%$ \\
$A<4 \mathrm{~m} \mathrm{~s}^{-1}$ & $1.3 \%$ & $3.6 \%$ & $2.5 \%$ & $3.9 \%$ \\
$A<5 \mathrm{~m} \mathrm{~s}^{-1}$ & $1.3 \%$ & $3.6 \%$ & $3.5 \%$ & $6.0 \%$ \\
\hline
\end{tabular}




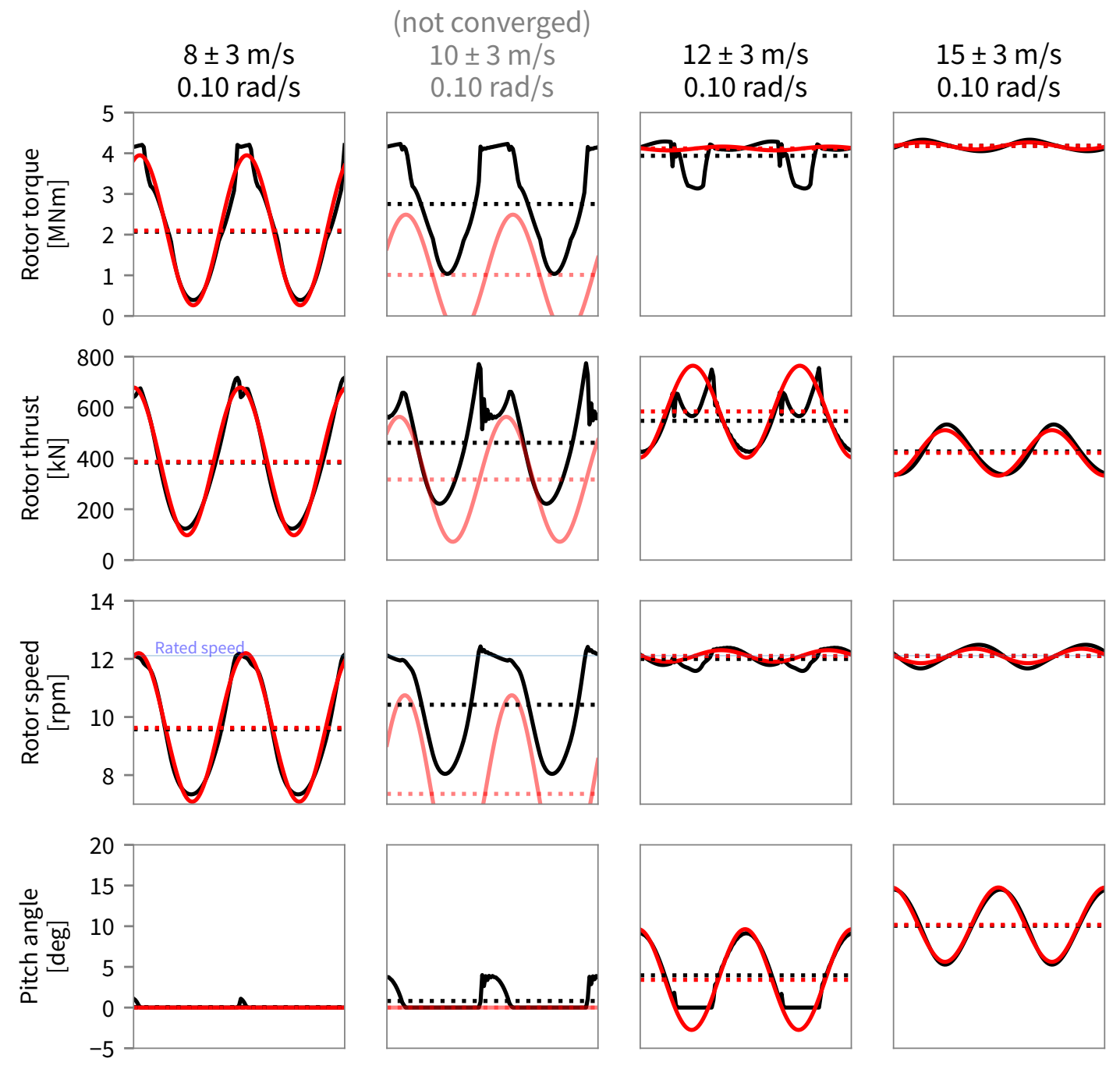

Figure C.13: Comparison of linearised and non-linear results for harmonic wind speed input varying at $0.10 \mathrm{rad} \mathrm{s}^{-1}$. Dotted lines show mean values. It was not possible to find a solution with a mean wind speed of $10 \mathrm{~m} \mathrm{~s}^{-1}$; see text for discussion. 

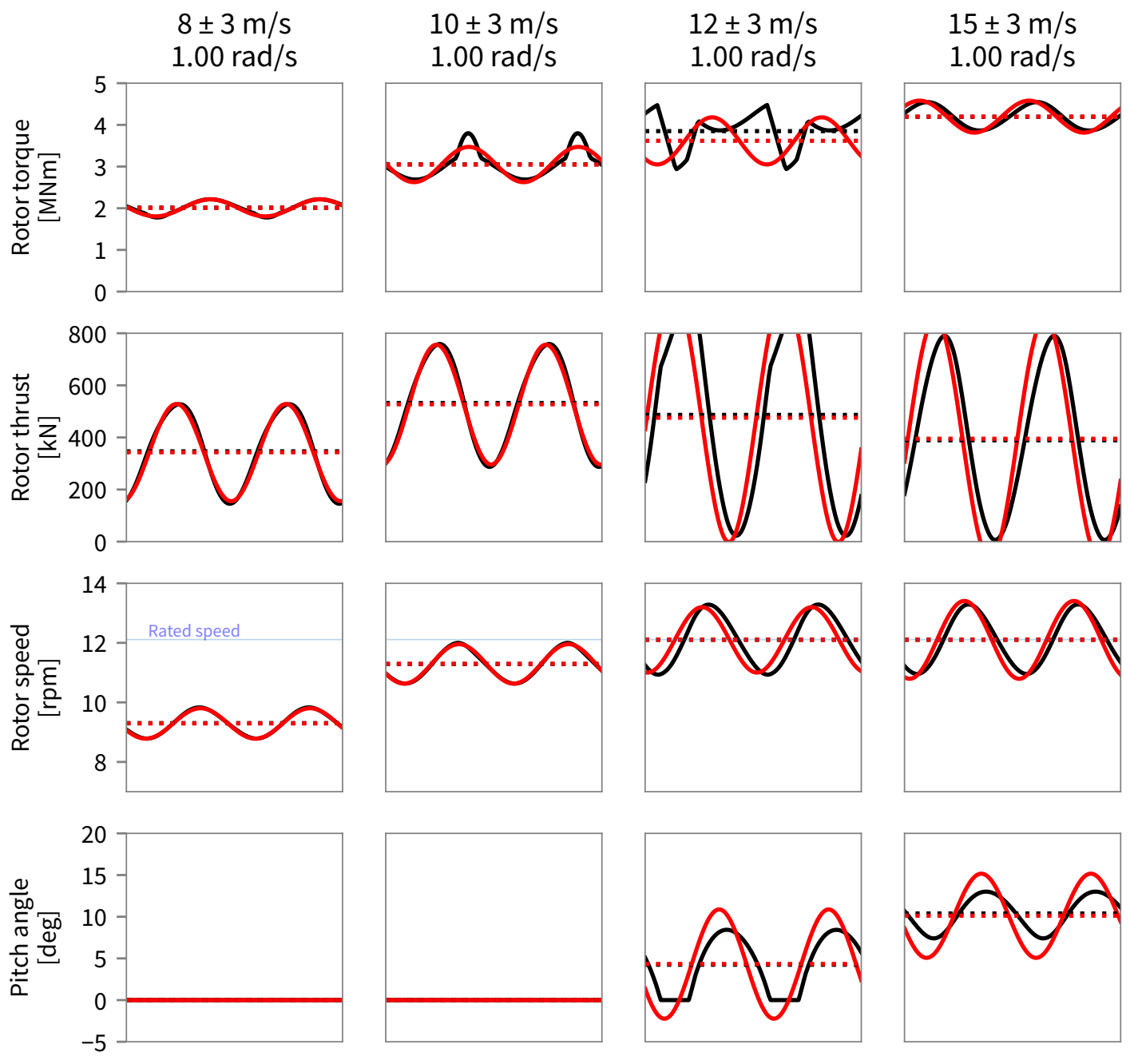

Figure C.14: Comparison of linearised and non-linear results for harmonic wind speed input varying at $1.00 \mathrm{rad} \mathrm{s}^{-1}$. Dotted lines show mean values. 